\title{
Messunsicherheit durch Simulation: Stand der Technik und neue Entwicklungen für die Koordinatenmesstechnik in der industriellen Produktion
}

\author{
Thomas Kistner ${ }^{1}$, Alessandro Gabbia ${ }^{1}$, Dietrich Imkamp ${ }^{1}$ \\ ${ }^{1}$ Carl Zeiss Industrielle Messtechnik $\mathrm{GmbH}$ \\ ZEISS Gruppe, 73446 Oberkochen, Deutschland
}

\begin{abstract}
Zusammenfassung
Die Messunsicherheitsermittlung durch Simulation - bekannt unter dem Begriff „virtuelles Koordinatenmessgerät" - ist eine hoch entwickelte aber nur im Bereich von Kalibrierlaboratorien verbreitete Methode. Durch die Weiterentwicklung des virtuellen Koordinatenmessgeräts soll diese Methode zusätzliche wesentliche Einflussgrößen berücksichtigen und im Hinblick auf ihre Handhabbarkeit für den industriellen Einsatz angepasst werden. Erste Vergleichsmessungen an einfachen Messaufgaben zeigen die Verbesserungen der Methodik. Für den industriellen Einsatz ist ein zweckmäßiger Kompromiss zwischen Simulationszeit und Stabilität der Ergebnisse zu finden. Durch die Beobachtung der Stabilität der Messunsicherheit während einer Simulation lässt sich eine Aussage darüber treffen, ab wann die Simulation einen stabilen Zustand erreicht hat.
\end{abstract}

Keywords: Fertigungsmesstechnik, Messunsicherheit, Monte-Carlo-Simulation,

\section{Einleitung:}

Koordinatenmesstechnik bezeichnet alle Messverfahren, welche die Koordinaten von Punkten im Raum erfassen. Die erfassten Koordinaten beziehen sich vorwiegend auf ein globales kartesisches Koordinatensystem, das durch den Aufbau der hier betrachteten Koordinatenmessgeräte verkörpert wird. Die Ausrichtung der Achsen dieses Koordinatensystems, oft auch als Gerätekoordinatensystem bezeichnet, orientiert sich an der Achsausrichtung des Koordinatenmessgeräts. Bei Messungen wird meist ein lokales Koordinatensystem am zu messenden Werkstück errichtet. Neben der vereinfachten Orientierung für den Bediener ermöglicht es die normgerechten Ausrichtung anhand von Bezugselementen der technischen Zeichnung des zu messenden Werkstücks (vgl. [1], S.260-262).

Das Ergebnis einer Messung dient als Grundlage für die Bewertung der Qualität des gemessenen Objekts. Hierbei wird in einem ersten Schritt der Messwert mit dem Sollwert verglichen. Der Messwert setzt sich aus dem wahren Wert sowie zufälligen und systematischen Messabweichungen zusammen. Zufällige Abweichungen rufen Variationen bei mehrmaligen Beobachtungen der Messgröße hervor. Die Korrektion dieser Komponente ist nicht möglich. Eine Vergrößerung der Beobachtungsanzahl liefert hier zusätzlichen Erkenntnisgewinn, um diesen Einflussfaktor zu verringern, was aber in der Koordinatenmesstechnik nicht üblich ist. Ein Teil der systematischen Abweichungen ist quantifizierbar. Der Einfluss dieser Komponenten auf das Messergebnis kann somit ausgeglichen werden. Die Aussage über die Qualität einer Messung steigt mit der Anzahl der erkannten Einflussparameter des Messergebnisses an. Es ist somit vorauszusetzen, dass ausreichend Informationen über den Messvorgang und die Einflussparameter auf diesen vorhanden sind. Anhand dieser Sammlung von Informationen kann der beste Schätzwert um seine Unsicherheit erweitert werden.

Die Kenntnis der Messunsicherheit und ihr Größenordnung spielt für Forschung und Industrie eine große Rolle. Durch Qualitätsrichtlinien wird die Kenntnis über die eigenen Prozesse und deren Fähigkeit gefordert. Hierbei ist die Mess-unsicherheit ein Indikator für die Beurteilung. Neben dem Nachweis der Eignung kann die Kenntnis der Messunsicherheit auch zur Prozessverbesserung genutzt werden.

\section{Das virtuelle Koordinatenmessgerät:}

Die Bestimmung der Messunsicherheit durch Simulation basiert auf einer umfassenden Modellierung des Messvorgangs [2]. Die Simulation löst Probleme unter Zuhilfenahme der Wahrscheinlichkeitstheorie. 
Mathematischer Hintergrund ist hierbei die Monte-Carlo-Simulation. Dafür erforderlich ist die Kenntnis über die Eigenschaften der einzelnen Parameter, deren Wahrscheinlichkeitsverteilung und Grenzen der Abweichungen [3]. In der Simulation werden
DOI 10.5162/sensoren2016/6.3.3

eine verlässliche Aussage über die Messunsicherheit treffen. Dieses Ergebnis ist abhängig von der Stabilität der Simulation und der Qualität der Beschreibung der Eingangsparameter.

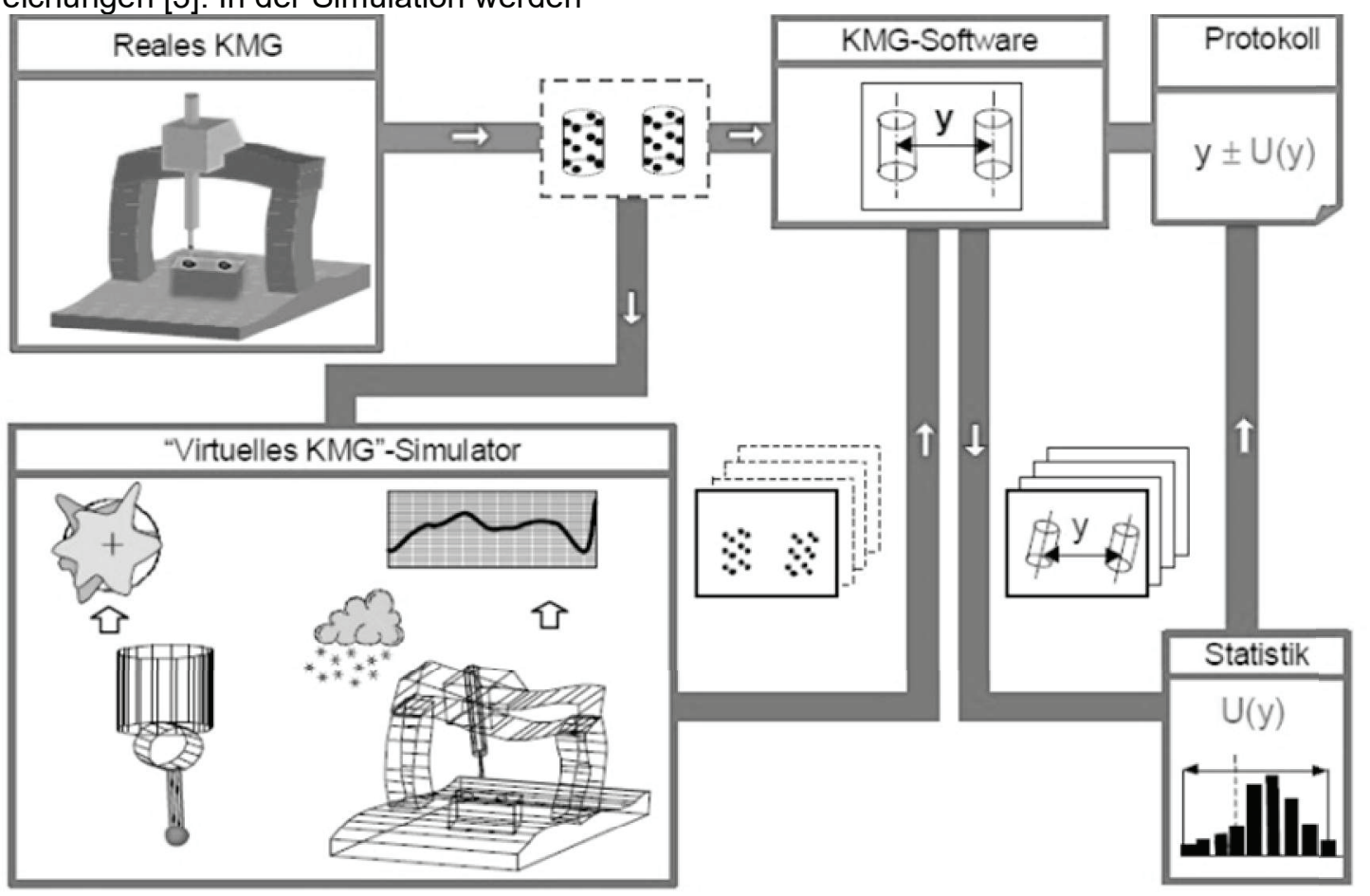

\section{Abb.. 1: Funktionsprinzip des virtuellen Koordinatenmessgeräts [4]}

diese Werte, unter Zuhilfenahme von Zufallszahlen, nach jeweiliger Wahrscheinlichkeit verteilt, innerhalb ihrer Grenzen variiert (vgl. [2], S.9-11).

Durch eine ausreichend hohe Anzahl an Simulationsschritten ergibt sich ein aussagekräftiges Ergebnis, welches eine zuverlässige Aussage über die Messunsicherheit der betrachteten Geometrie zulässt. Die Umsetzung dieser Methodik auf Koordinatenmessgeräten findet durch das VCMM (virtual coordinate measuring machine - deutsch: virtuelles Koordinatenmessgerät) statt [5]. Aufgrund der umfassenden Eingangsparameter in die Simulation werden fortgeschrittene Kenntnisse über das Verhalten von Koordinatenmessgeräten vorausgesetzt.

Abbildung 1 veranschaulicht das Funktionsprinzip des virtuellen Koordinatenmessgeräts. Die Simulation wird dabei separat zur Messung im Anschluss daran durchgeführt. Die Integration in vorhandene Messabläufe ist dadurch gegeben. Die Messpunkte werden zusammen mit der Tastercharakteristik, dem Restfehlerfeld des Koordinatenmessgeräts und weiteren Parametern an die Simulation übergeben. Mit jedem Schritt der Simulation wird der Messvorgang mit den dafür "gewürfelten“ Parametern simuliert. Nach einer ausreichend großen Anzahl an Wiederholungen kann man
Die erste Generation des virtuellen Koordinatenmessgeräts wurde 2002 fertiggestellt [6]. Diese Implementierung hatte eine große Bedeutung für die Koordinatenmesstechnik. Im Gegensatz zu bisherigen Methoden konnten die Parameter exakt auf das verwendete Gerät angepasst werden. Dem damaligen Stand der Technik entsprechend wurde die Punkterfassung durch Einzelpunkte unterstützt. Durch Fortschritte in der Koordinatenmesstechnik sind Weiterentwicklungen entstanden, welche eine Anpassung des virtuellen Koordinatenmessgeräts an den heutigen Stand der Technik fordern. Diese Anpassungen werden in der Weiterentwicklung unter dem Arbeitstitel VCMM II zusammen mit den Projektpartnern der physikalisch-technischen Bundesanstalt (PTB), Hexagon Metrology $\mathrm{GmbH}$, feinmess und eumetron vorgenommen.

\section{Einflussgrößen:}

Auf der nachfolgenden Seite werden in Abbildung 2 die Einflussgrößen des virtuellen Koordinatenmessgeräts in einem IshikawaDiagramm dargestellt. Hierbei wird sich auf die durch den Anwender eingebbaren Einflussgrößen der Monte-Carlo-Simulation des VCMM II beschränkt. Die Neuheiten des VCMM II in Bezug auf vorherige Entwicklungen werden herausgestellt. Es erfolgen weitere Differenzierungen hinsichtlich der Häufigkeit der Eingabe und dem Zeitpunkt der Erfassung von Einflussgrößen. 


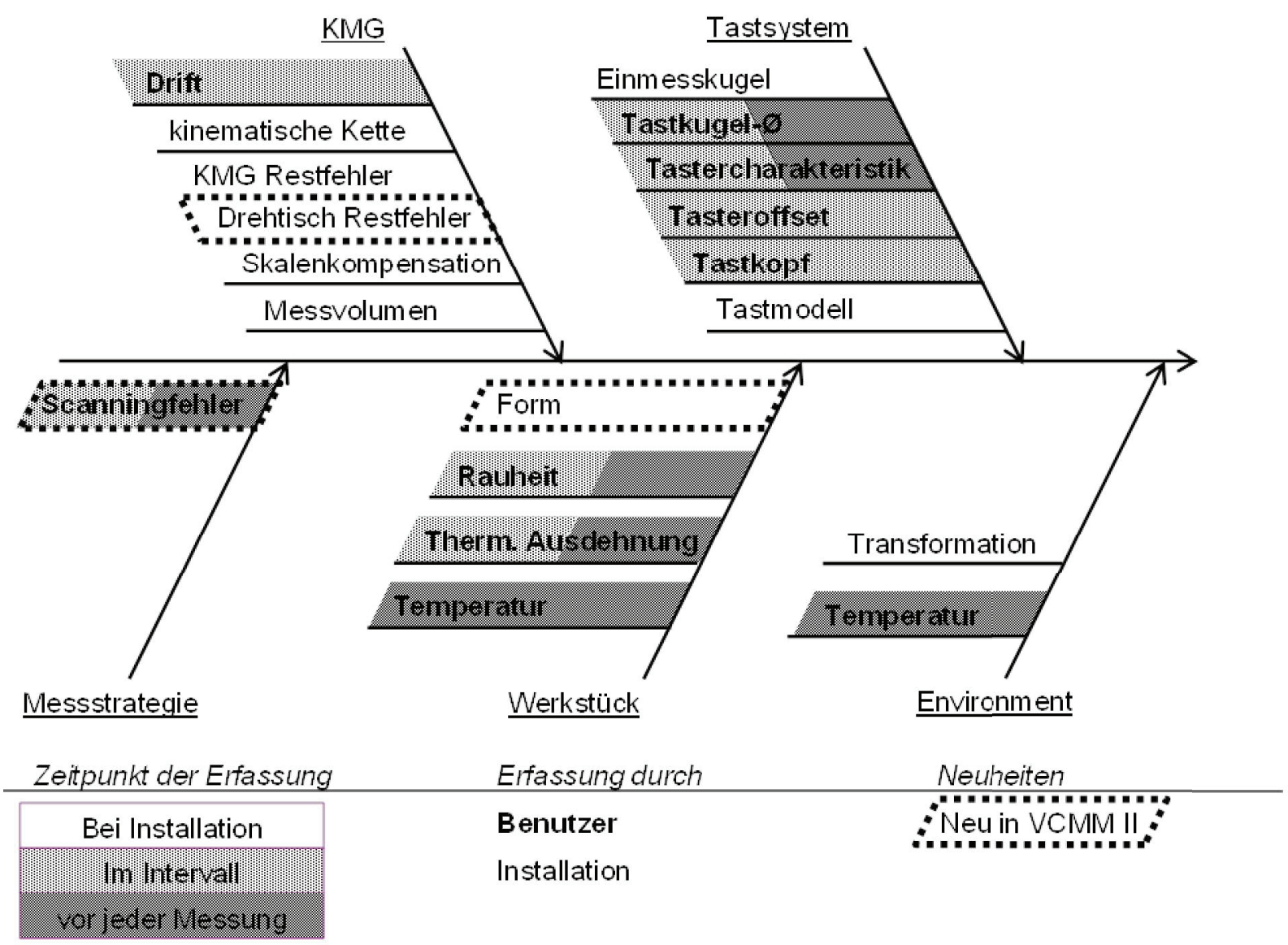

\section{Abb.. 2: Durch Anwender eingebbare Eingangsgrößen}

Die Einflussgrößen werden in fünf Zweige unterteilt. Da es sich hierbei um die von Anwender einzugebenden Parameter handelt, wird auf die Einflussgröße „Mensch“ verzichtet. Stattdessen wird der Zweig "Maschine" in Koordinatenmessgerät, kurz KMG, und Tastsystem unterteilt.

Die bedeutendste Erweiterung des VCMM II ist die Berücksichtigung des Scannings bei der Messunsicherheitsberechnung. Für viele Messaufgaben konnte die Messunsicherheit bisher nicht berechnet werden, da der Vorgänger VCMM I nur bei Messungen mit Einzelpunktaufnahme funktioniert. Durch die Erweiterung der Methodik für Scanningmessungen erfolgt eine Anpassung an den Stand der Technik. Der entstehende dynamischen Einfluss macht eine Anpassung der Einmessroutine erforderlich. Eine zusätzliche Erweiterung stellt die Berücksichtigung der Form des Messelements dar. Hierfür werden die Einflüsse des Fertigungsprozesses auf das Werkstück beschrieben. Dies geschieht durch die Durchführung einer Referenzmessung in Anschluss an die Standardmessung. Die Messstrategie dieser Referenzmessung zeichnet sich durch eine höhere Punktedichte in Kombination mit einer geringeren Messgeschwindigkeit aus.
Durch diese optimierte Messung können insbesondere Formabweichungen und Abweichungen aus dynamischen Messeinflüssen erkannt und quantifiziert werden.

Der Aufwand für die Beschreibung der Eingangsgrößen und die damit verbundenen Kosten sind bisher eine große Herausforderung für potentielle Anwender. Aus diesem Grund wird es im VCMM II für die meisten Eingangsgrößen mindestens zwei Eingabemöglichkeiten geben. Diese unterscheiden sich in der Genauigkeit der Beschreibung. Die hochgenaue Beschreibung erfordert einen hohen Zeitaufwand, wird jedoch ein exaktes Ergebnis liefern. Diese Art der Beschreibung wird Laboratorien und zentralen Messräumen von Unternehmen empfohlen. Die weniger genaue Beschreibung der Eingangsgröße bedeutet einen geringeren Zeit- und Kostenaufwand, das Ergebnis weist jedoch einen größeren Unsicherheitswert auf. Je nach Anwendungsfall und Größenordnung des Tolerierungswerts kann diese Art der Beschreibung ausreichend sein. Bei groben industriellen Fertigungsverfahren oder relativ großen Toleranzen können diese Parameter für die Messunsicherheitsermittlung verwendet werden. Um ein aussagekräftiges Ergebnis zu erlangen, erfolgt diese Berechnung mit einer ausreichenden Anzahl an Iterationsschritten. 
Die Ergebnisse werden in einer Protokollstatistik dargestellt. Diese soll eine Übersicht über die einzelnen Unsicherheitsbeiträge enthalten und ermöglicht so eine Analyse der einzelnen Beiträge. Darauf aufbauend kann eine Optimierung des Messprogramms hinsichtlich der einzelnen Einflüsse erfolgen. Die Option VCMM /I soll zukünftig in die Bediensoftware des Koordinatenmessgeräts integriert werden.

\section{Stabilität der Messunsicherheitssimulation}

Die Monte-Carlo-Simulation liefert nur dann eine zuverlässige Aussage, wenn das Ergebnis als ausreichend stabil betrachtet wird. Nach GUM Supplement 1 liefert ein Wert ab $10^{\wedge} 5$ bis $10^{\wedge} 6 \quad$ (vgl. [2], S.29) Wiederholungen zuverlässig ein Überlappungsintervall von 95\%. Durch die Komplexität der Berechnungen und die damit verbundene Rechenzeit ist diese Anzahl an Wiederholungen für den Einsatz des virtuellen Koordinatenmessgeräts nicht praxisgerecht. Um trotzdem ein aussagekräftiges Ergebnis zu erhalten, wird die adaptive Methode der MCSimulation (vgl.[2], S.33) angewandt. Dafür wird ein relatives Stabilitätskriterium eingeführt, welches die Änderung der Varianz betrachtet. Das Kriterium beschreibt einen Grenzwert, ab dessen Unterschreitung das Ergebnis der Simulation als stabil betrachtet wird.

Der Transfer dieser Methode von der Theorie in die Praxis zeigt, dass schon ab einer relativ geringen Anzahl an Simulationen die Werte ausreichend stabil werden. In Abbildung 3 wird der Verlauf der berechneten Messunsicherheit einer Messunsicherheitssimulation in Abhängigkeit von der Anzahl der Simulationen dargestellt. Dieser Verlauf ist typisch für die Abhängigkeit der Messunsicherheit von der Anzahl der Simulationen.
DOI 10.5162/sensoren2016/6.3.3

Die maximale Anzahl an Simulationen wurde hierbei auf 100.000 festgelegt, um die Forderungen des GUM bezüglich der Mindestzahl an Wiederholungen zu erfüllen und somit ein statistisch stabiles Ergebnis zu erlangen.

Der Ergebnisverlauf zeigt innerhalb der ersten 250 Wiederholungen große Schwankungen der Messunsicherheit. Dieses Verhalten ist zu erwarten gewesen, da bei einer geringen Anzahl an Wiederholungen der Einfluss einzelner Zufallsereignisse der Simulation signifikant sein können.

Ab 250 Wiederholungen nähert sich die Messunsicherheit dem zu erwartenden Ergebnis an. Die Differenzen zum Grenzwert bei 100.000 Simulationen betragen weniger als $4 \%$ und verringern sich im Lauf der Simulation mit wachsender Anzahl an Wiederholungen. $A b$ der 1000. Wiederholung beträgt der Unterschied der Messunsicherheit weniger als $1 \%$ zu der als verlässlich definierten Messunsicherheit. Ab 5000 Wiederholungen liegt die Differenz sogar deutlich unter 0,5\%.

Durch die Betrachtung der Änderung der Messunsicherheit im Verlauf einer Simulation wird deutlich, dass bei dieser Betrachtung stabile Ergebnisse mit deutlich weniger Simulationsdurchläufen zu erwarten sind. Um eine Unterschätzung der Messunsicherheit zu vermeiden, kann man bei der Verwendung eines Simulationsergebnisses mit einer geringen Anzahl an Wiederholungen die Verwendung eines Sicherheitsaufschlags von wenigen Prozent der simulierten Messunsicherheit in Betracht ziehen. Da die Schwankungen der Messunsicherheit von den Eingangsparametern der Simulation abhängig sind, ist auch die Einführung eines relativen Stabilitätskriteriums denkbar.

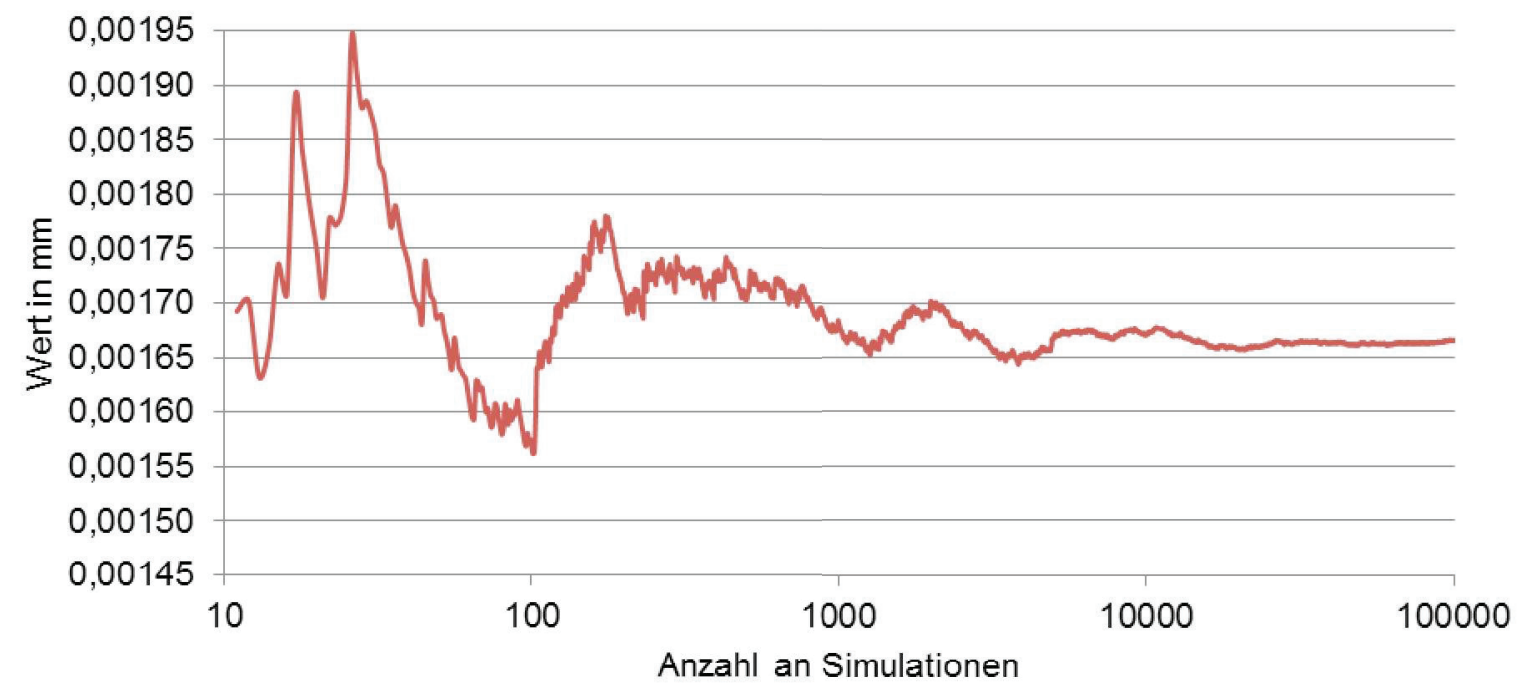

Abb.. 3: Verlauf der Messunsicherheit einer Messunsicherheitssimulation 


\section{Berechnung der Messunsicherheit:}

Um verschiedene Arten der Messunsicherheitsermittlung zu vergleichen, wurde eine einfache Messaufgabe definiert und anhand verschiedener Methoden die Messunsicherheit ermittelt. Als Grundlage dient jeweils die Messung des Durchmessers eines Werkstück mit dem Nennradius von 0,937 mm. Das Werkstück wurde vor der Durchführung der Messung in einem DAkkSakkrediterten Kalibrierlabor auf einem Koordinatenmessgerät kalibriert. Bei der Kalibriermessung im Einzelpunkmodus wurden 50 gleichverteilte Messpunkte auf einer vorab definierten Messebene aufgenommen. Das Ausgleichselement wurde als Gaußelement berechnet. Der Durchmesser der Antastkugel ist mit 0,3 mm definiert. Im Anschluss an die Kalibrierung wurde das Werkstück auf einem Koordinatenmessgerät gemessen. Um die Vergleichbarkeit zwischen Kalibrierergebnis und Messergebnis zu gewährleisten, wurden hierbei die gleichen Mess- und Auswerteparameter angewandt. Ergebnisse der Messungen mit der zugeordneten erweiterten Messunsicherheit werden in Abbildung 4 dargestellt. Die Messunsicherheit der Einzelpunktmessungen wird jeweils durch das VCMM I bestimmt. Bei der Scanningmessung fand die Berechnung durch eine Messunsicherheitsbilanz nach VDI/VDE 2617-11 [7] und durch das VCMM II im Entwicklungsstand statt.
DOI 10.5162/sensoren2016/6.3.3 Die Ergebnisse der durch verschiedene Messmethoden ermittelten Durchmesser sind untereinander vergleichbar. Die ermittelten Messunsicherheiten weisen jedoch große Unterschiede auf.

Hierbei sind die auftretenden Unterschiede zwischen Messunsicherheitsbudget, mit einer erweiterten Messunsicherheit $U=10,7 \mu \mathrm{m}$, und VCMM I hinreichend bekannt und nachgewiesen [8]. Differenzen zwischen den nach VCMM I ermittelten Messunsicherheiten sind ebenso erkennbar. Aufgrund auftretender Schwankungen bei mehrmaliger Ermittlung der Messunsicherheiten mit dem VCMM I wird bei Kalibriermessungen oftmals ein Aufschlag auf die Unsicherheit gegeben. Dadurch wird bei der Durchführung mehrerer Messung die Ausgabe einer gleichbleibenden erweiterten Messunsicherheit, in diesem Fall $3 \mu \mathrm{m}$, ermöglicht. Die im Rahmen der Messreihe ermittelte erweiterte Messunsicherheit mittels VCMM I weist mit knapp $2 \mu \mathrm{m}$ einen geringeren Wert auf. Das Messunsicherheitsergebnis des VCMM II ist mit $U=6 \mu \mathrm{m}$ doppelt so groß wie die Unsicherheit der Kalibrierung. Dieser Unterschied ist durch die Berücksichtigung weiterer Einflüsse erklärbar. Weiterhin sind im derzeitigen Entwicklungsstand des VCMM II einige Eingangsparameter bisher nur grob beschrieben und wirken sich dadurch ebenso auf die Größenordnung der ermittelten Messunsicherheit aus.

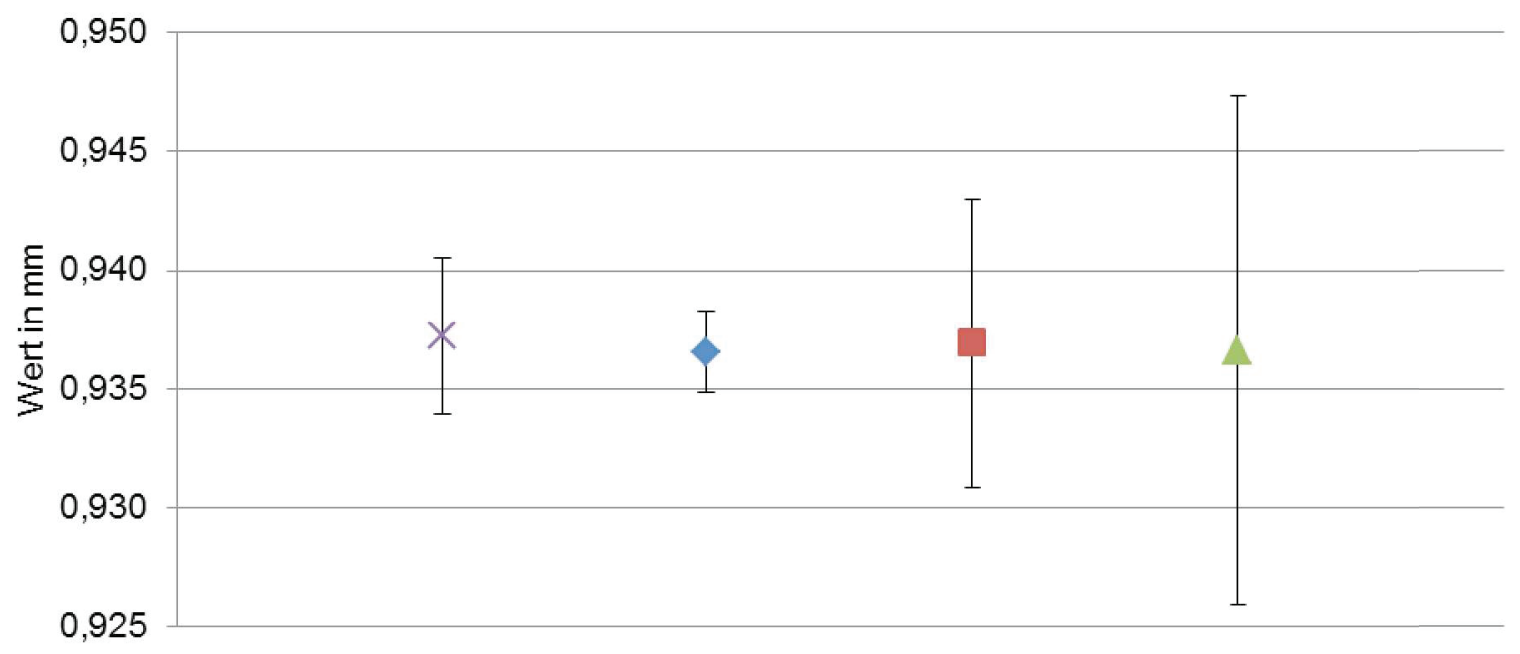

\begin{tabular}{|c|c|c|}
\hline Messgerät: & \multicolumn{2}{|l|}{ Koordinatenmessgerät } \\
\hline Art der Punkterfassung: & Einzelpunkt & Scanning \\
\hline \multirow[t]{2}{*}{ Allgemein: } & Anzahl der Messpunkte: 50 & Tastkugeldurchmesser: 0,3 mm \\
\hline & Filter: Gaußfilter & Ausgleichselement: Gaußelement \\
\hline
\end{tabular}

\section{Abb.. 4: Vergleich verschiedener Methoden}

zur Messunsicherheitsermittlung 
Da neben diesen Methoden zur Messunsicherheitsermittlung noch weitere Methoden existieren, welche bei vergleichbaren Eingangsparametern verschiedene Ergebnisse liefern, wäre ein Vergleich der verschiedenen Methoden und eine Aussage über die Qualität der Messunsicherheitsermittlung interessant. Ein Ansatz hierbei kann die Anwendung von [9] mit der Berücksichtigung von [10] darstellen. Durch die systematische Aufbereitung einzelner Unsicherheitsbeiträge der verschiedenen Methoden können die Unterschiede herausgearbeitet werden. Problematisch ist dies jedoch bei der Messunsicherheitsermittlung durch Simulation. Für einen systematischen Vergleich der Methoden untereinander stellt das derzeitige „Black-BoxModell" ein Hindernis dar, da dem Anwender die Größenordnung der einzelnen Unsicherheitsbeiträge nicht angezeigt wird. Dies steht einer Vergleichbarkeit der Methoden untereinander im Weg. Durch die geplante Darstellung der einzelnen Einflussgrößen kann ein Vergleich nach bestehender Methodik erfolgen.

\section{Ausblick}

Die Forderungen der Normenreihe ISO 14253 und ISO 9001 zur Angabe der Messunsicherheit werden mit VCMM II erfüllt. Das VCMM II dient dem Anwender als Werkzeug und Hilfsmittel für deren Bestimmung. Die Anpassungsfähigkeit des VCMM II an die jeweilige Messaufgabe ermöglichen den Einsatz bei Prüfprozessplanungen von Serienmessungen als auch bei hochgenauen Untersuchungen im Prototypenbau.

\section{Literaturnachweis}

[1] Weckenmann, A. (Hrsg.): Koordinatenmesstechnik: Flexible Messstrategien für Maß, Form und Lage; 2. Aufl. München : Hanser Verlag, 2012. - ISBN 978-3-446-40739-8

[2] JGCM 101:2008: Evaluation of measurement data - Supplement 1 to the "Guide to the expression of uncertainty in measurement" Propagation of distributions using a Monte Carlo method, JGCM (Joint Committee for Guides in Metrology), 2008. (Internet: http://www.bipm.org/en/publications/ guides/gum.html)

[3] Weckenmann, A.; Sommer, K.-H.; Siebert B. R L.: Qualität für Messergebnisse Messunsicherheit nach GUM, in: tm Technisches Messen, Oldenbourg Industrieverlag, 73 (2006) 4, S. 189-199

[4] VCMM Nutzerhandbuch, PhysikalischTechnische Bundesanstalt, Stand 2014

[5] Wäldele, F. und Schwenke, H.: Automatische Bestimmung der Messunsicherheiten auf KMGs auf dem Weg in die industrielle Praxis, in: tm Technisches Messen, Oldenbourg Industrieverlag, 69 (2002) 12, S. 550-557

[6] Trapet, E.; et.al.: Development of Methods and Guidelines to Establish Traceability for

Measurements with Coordinate Machines; Final Report; physikalisch-technische Bundesanstalt; 1998

[7] VDI/VDE 2617 Blatt 11 - Genauigkeit von Koordinatenmessgeräten - Kenngrößen und deren Prüfung - Ermittlung der Unsicherheit von Messungen auf Koordinatenmessgeräten durch Messunsicherheitsbilanzen, März 2011

[8] Hernla, M.; Franke, M.; Wendt, K.: Aufgabenspezifische Messunsicherheit von Koordinatenmessungen. tm - Technisches Messen, Oldenbourg Industrieverlag 77 (2010) 11, S. 607-615

[9] DIN EN ISO 14253-3:2011-10 Geometrische Produktspezifikation (GPS) - Prüfung von Werkstücken und Meßgeräten durch Messen Teil 3: Richtlinien für das Erzielen einer Einigung über Messunsicherheitsangaben (ISO 14253-3:2011); Deutsche Fassung EN ISO 14253-3:2011, Oktober 2011

[10] DIN EN ISO 14253-2:2011-10 Geometrische Produktspezifikation (GPS) - Prüfung von Werkstücken und Meßgeräten durch Messen Teil 2: Anleitung zur Schätzung der Unsicherheit bei GPS-Messungen, bei der Kalibrierung von Messgeräten und bei der Produktprüfung (ISO 14253-2:2011); Deutsche Fassung EN ISO 14253-2:2011, Oktober 2011 